\title{
Theoretical analysis of JIT elements for implementation in maintenance sector
}

\author{
Sandeep Phogat ${ }^{a^{*}}$ and A. K. Gupta ${ }^{\text {b }}$
}

\begin{abstract}
${ }^{a}$ Assistant Professor, Mechanical Engineering Department, Amity University Haryana, Gurgaon, India ${ }^{b}$ Associate Professor, Mechanical Engineering Department, DCRUST Murthal, Sonipat, India

\begin{tabular}{l}
\hline C H R O N I C L E \\
\hline Article history: \\
Received July 2, 2016 \\
Received in revised format \\
September 10, 2016 \\
Accepted January 92017 \\
Available online \\
January 222017 \\
\hline Keywords: \\
Just in time (JIT) \\
Maintenance \\
JIT elements \\
JIT in maintenance \\
JIT in manufacturing
\end{tabular}

Just in time (JIT) philosophy has grown to a new high level since its evolution and has successfully been implemented in manufacturing. As it is universally accepted that present study is to evaluate various elements of JIT to implement in maintenance sector which have high degree of importance in Global context. It is because now maintenance also plays manufacturint role in optimization of business processes. Maintenance operations are much like to create the end products. JIT focuses on the processes, not the product. It can, therefore, be applied to any process in manufacturing or maintenance operations. For this, literature related JIT usage and performance is reviewed. Thirty-eight elements are analyzed from sixty-five research papers of global context. With aid of theoretical analysis and brain storming with maintenance specialists of JIT elements who have implemented it for manufacturing industries, eighteen elements are selected to check the implementation of JIT philosophy in maintenance in the article.
\end{abstract}

\section{Introduction}

Manufacturing companies are extremely competitive in today's fast-changing market. To manage this challenge, companies are searching for new ways to optimize their manufacturing practices. During the past decades, adopting Just in Time (JIT) production principles has been widely popularized to increase profitability and productivity. JIT ideology makes the manufacturing flow more responsive to instability and apparatus failures (Faria \& Nunes, 2012). However, most of the organizations are solely concentrating on manufacturing effectiveness through utilization of JIT, and one requirement for the success of a JIT in manufacturing is the adoption of JIT in maintenance. High dependability is important for a JIT manufacturing system (Faria \& Nunes, 2012), but this requires effective maintenance and a systematic approach to avoid failures and organizing doubts (Johannesson et al., 2013). Risk assessment during the design of production systems and planning of maintenance activities is importance to make sure about the safety and health of maintenance workers.

\footnotetext{
* Corresponding author

E-mail address: sandeepphogat1@gmail.com (S. Phogat)

C 2017 Growing Science Ltd. All rights reserved.

doi: $10.5267 /$ j.uscm.2017.1.003
}

(C) 2017 Growing Science Ltd. All rights reserved. 
JIT in maintenance is the submission of JIT principle in maintenance environments. JIT system recognizes seven types of waste in maintenance. They are over production, waiting, process waste, transportation, defects, motion and inventory. These wastes are identified and efforts are made for the continuous improvement in process by eliminating the wastes. Thus, JIT in maintenance leads to maximize yield, profitability and productivity. JIT in maintenance is basically tools reliability focused and reduces need for maintenance troubleshooting and repairs. JIT in maintenance protects equipment and system from the root causes of malfunctions, failures and downtime stress. From the sources of waste uptime can be enhanced and cost can be lowered for maintenance. The present learning aims to study the JIT elements of manufacturing with a view of application in maintenance.

\section{Literature Review}

Reda (1987) reviewed research papers on "Kanban" and briefed JIT with its concepts. As a conclusion, Kanban contrasted other approaches of production and inventory control. Sewell (1990) evaluated validity model of hybrid manufacturing management in combination with JIT and existing MRP operations by comparing with the conceptual approach based on systems engineering principles. Ebrahimpour et al. (1993) investigated the adaption of JIT in US industry. Ramarapu et al. (1994) contradicted the theory of JIT implementation of many researchers who believed that elimination of waste and production strategy are the most specific critical factors. Gelinas et al. (1996) presented some facts on buyer expectations, benefits, and success factors concerning JIT purchasing. Partnership strategies in suitability with JIT purchase was demonstrated and the favorable situations were also presented. Gunasekaran and Lyu (1997) described the effects of JIT on all operations of a firm, including design, accounting, finance, marketing, distribution, etc., and stated JIT as interest to all levels of a firm's management as it had been successful in large-scale manufacturing companies worldwide. They also aimed to motivate small scale and medium industries. Sakakibara et al. (1997) included JIT practices with infrastructure to develop a JIT model for effectiveness and performance study. They concluded that there exists no significant relationship between alone JIT practice \& manufacturing performance; there exists a strong bond between infrastructure \& JIT practices; manufacturing performance is dominating combination of infrastructure practice with JJIT management; infrastructure, by itself, is sufficient to explain manufacturing performance and (5) manufacturing performance is related to competitive advantage. McLachlin (1997) examined the necessity of management initiatives in successful implementation of JIT in manufacturing. Their case study based research showed that four out of six considered management initiatives supported the proposed theory. The supporting initiatives are employee responsibility, training, teamwork and commitment. As a conclusion they stated that employee involvement plays a central role in JIT implementation. Mahadevan (1997) classified 43 organizations into groups. The classification criteria were, ownership pattern, sector of industry, comfort \& size and anxiety factors. Lorefice (1998) explored the strategies and effect of cultural factors in successful implementation of JIT in Japanese Industry. They summarized flexibility, small lot size, elimination of wastes as major factors. They also extended their study to the resources supply and relationship between managers and suppliers. Gelinas (1999) described JIT as a waste elimination philosophy with a goal as problem solver. The firm wants implementation of JIT to be successful in one attempt, but are also concerned about cost and time of implementation.

Canel et al. (2000) stated that JIT gained strong toehold in industrial sector as a formal process to reduce waste while service sector still needs to be worked on for JIT benefits. Nature of JIT is process focus, and hence can be smeared to any industrial problem. They investigated JIT principles and presented a JIT framework for JIT in service industry. Chung et al. (2001) provided comprehension of prerequisites and JIT concept for application in public and private sector. Despite being so advantageous, they argued successful application of JIT in government organizations and exploration of JIT for operation management. Fullerton et al. (2001) collected 95 responses of resurvey from executives of JIT-practicing firms to superior understand the profit those firms have gain through JIT 
implementation. White et al. (2001) discussed the significance of integration of customer service and decision-making process of manufacturing firms in today's global market and environment to enhance the development for their competitive advantage. Kaynak (2002) studied JITP techniques and their relationship to firm performance. Supplier value addition and delivered quantities are the two major positive techniques related directly to the performance of the firm. They concluded that commitment of top management to JITP and other techniques affect changes in the organization. Low et al. (2003) identified similarities between construction setting and these concepts and subsequently integrating them. By such integration, the JIT system can be achieved without expending additional resources. A study by Hopp et al. (2003) shows that, the progressive simplification by literature experts has brought the language to a level wherein misunderstanding has appeared in defining pull. To reduce the confusion, generalized precise definition was given as, an essential mechanism to limit WIP \& lean, which fundamentally is about to minimize the variable buffering cost. Yang and Pan (2004) explained JIT production crucial role in supply chain management in manufacturing systems and companies are using it to gain and maintain a competitive advantage. This study presented an integrated inventory model to minimize cost and quality improvement investment by simultaneously optimizing the order quantity, lead time, process quality and number of deliveries. Yasin et al. (2004) investigated several effects of the execution of JIT using a two-tiered experimental approach that included mailed survey and field study and indicated that all the association types had enacted operational and procedural modifications in hold up of their JIT implementations. Salaheldin (2005) described the major individual modification prior to JIT accomplishment in Egyptian manufacturing firms, to identify the problems that these industries typically encounter in implementing it and investigated the connection between human modification efforts. Phan et al. (2005) empirically examined the output of JIT practices on financial, time and flexibility performances in Japan and the regression model applied showed that these had been significantly related. Statistical results also showed that, in Japanese manufacturers recently, the stable master schedule, setup time reduction and equipment layout play important roles in reducing cycle time and manufacturing cost, and increasing volume flexibility and inventory turnover.

Wakchware et al. (2006) described JIT literature from Indian manufacturing organizations and highlighted its elements and implementation issues. A survey was conducted in 45 industries to compare Japanese and Indian manufacturing philosophies and found practicing for, lot size reduction; respect for humanity; product scheduling; quality circles, lifelong employment; set up time reduction; kaizen; WIP reduction; preventive maintenance; smooth built up rate; and reliable and prompt delivery. Finally, it was concluded that the reason listed for slow execution of JIT are, lack of information; lack of justification for practicing JIT; lack of consultancy assistance available; lack of formal cross training program for workers; problems in maintenance time reduction; and initial high investment in layout reconfiguration to suit JIT requirements. Wong et al. (2006) studied the use of fuzzy set theory and genetic algorithms to generate JIT fabric-cutting schedule in a dynamic and fuzzy cutting environment. Experimental results demonstrated that the genetically optimized schedules get better the internal fulfillment of downstream manufacturing departments and decreased the production cost, simultaneously. Younies et al. (2007) discussed appliance of JIT in manufacturing industry and its advantages and disadvantages keeping in mind the Kanban system and cost accounting implications associated with JIT. Finally, the use of JIT strategy was cross examined, and findings were reported on its future advantages in the U.S. Sendil Kumar et al. (2007) described JIT philosophy, the method involved in the pull and push system; along the significance of measure of performance (MOP) and blocking mechanism in the Kanban system with its application with respect to JIT-KANBAN. Sandanayake et al. (2008) used modeling software and simulation technique to simulate and model perspective of selected main JIT variables. Mathematical model output highlighted the importance of JIT elements in the human operated mixed model assembly lines. Moreira et al. (2008) described JIT system implementation perception and its potential benefits by Portuguese manufacturing firms' managers. The findings showed that firms view JIT system as a method to eliminate waste, to reduce inventories and increase quality. Despite this good perception, very few firms have the essential conditions to productively implement a JIT production system. The study concluded that, the firms 
should use JIT as a philosophy rather than a solution for operations-related problems. Obamiro (2009) explored the association between JIT technique and industrialized performance of few selected Nigerian industries and the results enlighten that, there was an important relationship flanked by JIT practices and total quality management. These results demonstrated that JIT practices can be successfully implemented if certain supporting infrastructures are provided and they should be practiced at all levels and departments of an organization, rather than viewing it strictly for shop floor workers. Meybodi (2009) studied differences among traditional and JIT companies in selecting benchmarking output measures at different levels of organization. A range of industries like fabricated metal, electronics, communication, automotive, toots, rubber, chemicals, and paper products were examined. The study concluded that JIT companies are supplementary consistent in selecting benchmarking output measures that are aligned with organizational strategy. Deshmukh and Garg (2010) carried out a survey of JIT purchasing elements in Indian context and found out the relative importance of such elements. With the help of this survey some research directions were identified which needed close scrutiny. Mackelprang and Nair (2010) used meta-analysis of correlations approach to examine the association between JIT industrialized performance and practices outcomes. Existence of positive relationship was found between JIT industrialized practices and aggregate performance. This study highlighted the practices that have the greatest impact on individual performance outcomes. Adeyemi (2010) focused on the adoption of JIT production system (JITPS) in manufacturing practices of the third world countries is moderately limited. Because of this, production processes here are slow, wasteful and characterized by avoidable complexities. On examination of a typical third world countryNigeria, it was concluded that larger companies adopt JIT method more while the relatively smaller ones are still not attentive of the existence of the technique. Kumar (2010) reviewed the literature of JIT philosophy based quality organizations in Indian perspective and presented its concepts, accomplishment strategies and advantages.

Singh et al. (2011) discussed the behavior of JIT purchasing in Indian circumstances and provided a proportional analysis of difference between JIT purchasing and conventional Indian purchasing system by highlighting the problems encountered in JIT purchasing implementation and benefits. Shah (2011) developed an integrated inventory model for supply chain of single buyer and single supplier and minimized total integrated cost of the system. The sensitivity analysis suggested that the unit assessment cost, weakening rate of units in account and stock parameters were the critical factors. Singh et al. (2011a) presented the spread of JIT objectives, movement, concepts, benefits, elements, execution and motivation of JIT production strategy in Indian context. Lohal (2011) composed data from thirtythree industries by the assist of questionnaire fill up, on the source of collected data critical elements were identified and checked the degree of difficulty and degree of importance in adoption of JIT system, solutions were also highlighted to resolve these problems. Pheng et al. (2011) examined the use of JIT strategy for air travel organization with emphasis on management and airports terminal buildings operations. The principles focused in this study were waste elimination, uninterrupted work flow, pull production system, total quality control, employee involvement, top management commitment, long term working associations with suppliers and continuous improvement. It was established that the businesses which used JIT principles, like Japanese companies, were able to struggle successfully in the global market. The study highlighted the strengths as well as areas for potential improvements in the airport through the appliance of JIT principles. Gupta (2011) developed a theoretical model based on the reviewed literature in the area of examination quality and JIT philosophy. The model was tested by using authentic data commencing service organizations to check the persuade of JIT on service industries and finally framework to hold up the JIT execution in service organizations was developed. Hou et al. (2011) explored to make use of a case study to current various issues adjoining accomplishment of JIT strategy for an automotive organization and indicated that JIT production system was useful, and operating JIT manufacturing system could show the way to many reward to the industries. Fateha et al. (2012) discussed execution of JIT manufacturing in details. JIT manufacturing system identifies the unknown problems in the important chain and reduces waste production while rising the throughout (Sales-Raw Material Cost). 
Table 1

Yields literature revealed JIT elements of manufacturing

\begin{tabular}{|c|c|c|c|c|c|c|c|c|c|c|c|c|c|c|c|c|c|c|c|}
\hline \multirow{2}{*}{ Author } & \multicolumn{19}{|c|}{ JIT Elements } \\
\hline & A & $\mathrm{B}$ & $\mathrm{C}$ & $\mathrm{D}$ & $\mathrm{E}$ & $\mathrm{F}$ & $\mathrm{G}$ & $\mathrm{H}$ & $\mathrm{I}$ & $\mathrm{J}$ & $\mathrm{K}$ & $\mathrm{L}$ & $\mathrm{M}$ & $\mathrm{N}$ & $\mathrm{O}$ & $\mathrm{P}$ & $\mathrm{Q}$ & $\mathrm{R}$ & $\mathrm{S}$ \\
\hline Kumar (2014) & $\sqrt{ }$ & $\sqrt{ }$ & $\sqrt{ }$ & $\sqrt{ }$ & $\sqrt{ }$ & $\sqrt{ }$ & $\sqrt{ }$ & $\sqrt{ }$ & $\sqrt{ }$ & $\sqrt{ }$ & $\sqrt{ }$ & $\sqrt{ }$ & $\sqrt{ }$ & $\sqrt{ }$ & $\sqrt{ }$ & $\sqrt{ }$ & $\sqrt{ }$ & $\sqrt{ }$ & $\sqrt{ }$ \\
\hline Khan (2014) & & $\sqrt{ }$ & & $\sqrt{ }$ & & & & & & $\sqrt{ }$ & & $\sqrt{ }$ & $\sqrt{ }$ & & & & $\sqrt{ }$ & & \\
\hline Rahmani et al. (2014) & & & & $\sqrt{ }$ & $\sqrt{ }$ & $\sqrt{ }$ & $\sqrt{ }$ & $\sqrt{ }$ & $\sqrt{ }$ & $\sqrt{ }$ & & $\sqrt{ }$ & $\sqrt{ }$ & & $\sqrt{ }$ & $\sqrt{ }$ & $\sqrt{ }$ & & $\sqrt{ }$ \\
\hline Kamboj et al. (2013) & & $\sqrt{ }$ & $\sqrt{ }$ & $\sqrt{ }$ & & & & $\sqrt{ }$ & $\sqrt{ }$ & & & $\sqrt{ }$ & $\sqrt{ }$ & & $\sqrt{ }$ & $\sqrt{ }$ & $\sqrt{ }$ & & \\
\hline Paliwal et al. (2013) & & $\sqrt{ }$ & $\sqrt{ }$ & $\sqrt{ }$ & & & $\sqrt{ }$ & & $\sqrt{ }$ & $\sqrt{ }$ & & $\sqrt{ }$ & & & $\sqrt{ }$ & & $\sqrt{ }$ & & \\
\hline Kootanaee et al. (2013) & & $\sqrt{ }$ & $\sqrt{ }$ & $\sqrt{ }$ & & & $\sqrt{ }$ & $\sqrt{ }$ & $\sqrt{ }$ & & $\sqrt{ }$ & $\sqrt{ }$ & & & & & & & \\
\hline Fateha et al. (2012) & & $\sqrt{ }$ & $\sqrt{ }$ & $\sqrt{ }$ & & & & & $\sqrt{ }$ & $\sqrt{ }$ & & $\sqrt{ }$ & & & $\sqrt{ }$ & & $\sqrt{ }$ & & $\sqrt{ }$ \\
\hline Gupta (2012) & & $\sqrt{ }$ & $\sqrt{ }$ & $\sqrt{ }$ & & & & & & & $\sqrt{ }$ & $\sqrt{ }$ & $\sqrt{ }$ & $\sqrt{ }$ & & $\sqrt{ }$ & & & \\
\hline Farsijani et al. (2012) & & & $\sqrt{ }$ & & & & $\sqrt{ }$ & & $\sqrt{ }$ & $\sqrt{ }$ & $\sqrt{ }$ & $\sqrt{ }$ & & & $\sqrt{ }$ & & $\sqrt{ }$ & & \\
\hline Gupta \& Garg (2012) & $\sqrt{ }$ & $\sqrt{ }$ & $\sqrt{ }$ & $\sqrt{ }$ & & & & & $\sqrt{ }$ & $\sqrt{ }$ & $\sqrt{ }$ & $\sqrt{ }$ & $\sqrt{ }$ & & & & & & \\
\hline Gupta (2012) & & $\sqrt{ }$ & & $\sqrt{ }$ & & & & $\sqrt{ }$ & & & $\sqrt{ }$ & $\sqrt{ }$ & & $\sqrt{ }$ & & & & & \\
\hline Fouad et al. (2012) & & $\sqrt{ }$ & $\sqrt{ }$ & $\sqrt{ }$ & & & & & $\sqrt{ }$ & $\sqrt{ }$ & $\sqrt{ }$ & $\sqrt{ }$ & & $\sqrt{ }$ & $\sqrt{ }$ & $\sqrt{ }$ & $\sqrt{ }$ & & \\
\hline Singh et al. (2012) & & $\sqrt{ }$ & $\sqrt{ }$ & $\sqrt{ }$ & & & & $\sqrt{ }$ & & & $\sqrt{ }$ & $\sqrt{ }$ & $\sqrt{ }$ & $\sqrt{ }$ & & & & & $\sqrt{ }$ \\
\hline Shah (2011) & & & & & & & & & & & & & $\sqrt{ }$ & & & & & & \\
\hline Singh et al. (2011) & $\sqrt{ }$ & $\sqrt{ }$ & $\sqrt{ }$ & & & & $\sqrt{ }$ & $\sqrt{ }$ & $\sqrt{ }$ & $\sqrt{ }$ & $\sqrt{ }$ & & $\sqrt{ }$ & & $\sqrt{ }$ & $\sqrt{ }$ & $\sqrt{ }$ & $\sqrt{ }$ & $\sqrt{ }$ \\
\hline Lohar (2011) & & $\sqrt{ }$ & & & & $\sqrt{ }$ & $\sqrt{ }$ & & $\sqrt{ }$ & & & $\sqrt{ }$ & & & $\sqrt{ }$ & $\sqrt{ }$ & $\sqrt{ }$ & & \\
\hline Singh (2011) & & & & $\sqrt{ }$ & & & $\sqrt{ }$ & & $\sqrt{ }$ & $\sqrt{ }$ & $\sqrt{ }$ & $\sqrt{ }$ & $\sqrt{ }$ & & & 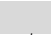 & 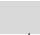 & & \\
\hline Gupta (2011) & $\sqrt{ }$ & $\sqrt{ }$ & $\sqrt{ }$ & $\sqrt{ }$ & & & & & $\sqrt{ }$ & & & $\sqrt{ }$ & $\sqrt{ }$ & & $\sqrt{ }$ & $\sqrt{ }$ & $\sqrt{ }$ & & \\
\hline Tewari et al. (2011) & & & $\sqrt{ }$ & $\sqrt{ }$ & $\sqrt{ }$ & $\sqrt{ }$ & & & & $\sqrt{ }$ & & $\sqrt{ }$ & & & & & & & $\sqrt{ }$ \\
\hline Pheng et al. (2011) & & $\sqrt{ }$ & $\sqrt{ }$ & $\sqrt{ }$ & & & $\sqrt{ }$ & & & & & $\sqrt{ }$ & $\sqrt{ }$ & & & & & & \\
\hline Gupta (2011) & & $\sqrt{ }$ & $\sqrt{ }$ & $\sqrt{ }$ & $\sqrt{ }$ & & $\sqrt{ }$ & & $\sqrt{ }$ & & $\sqrt{ }$ & $\sqrt{ }$ & & $\sqrt{ }$ & $\sqrt{ }$ & & & & $\sqrt{ }$ \\
\hline Hou et al. (2011) & & $\sqrt{ }$ & $\sqrt{ }$ & $\sqrt{ }$ & & & & & $\sqrt{ }$ & & $\sqrt{ }$ & $\sqrt{ }$ & $\sqrt{ }$ & & & & & & $\sqrt{ }$ \\
\hline Agrawal (2010) & & $\sqrt{ }$ & $\sqrt{ }$ & $\sqrt{ }$ & $\sqrt{ }$ & & $\sqrt{ }$ & & & $\sqrt{ }$ & $\sqrt{ }$ & $\sqrt{ }$ & & & $\sqrt{ }$ & $\sqrt{ }$ & & & \\
\hline Mackelprang \& Nair & & & & & & & $\sqrt{ }$ & & & $\sqrt{ }$ & & $\sqrt{ }$ & $\sqrt{ }$ & & $\sqrt{ }$ & & $\sqrt{ }$ & & \\
\hline Adeyemi (2010) & & & $\sqrt{ }$ & $\sqrt{ }$ & & & & & $\sqrt{ }$ & & & $\sqrt{ }$ & $\sqrt{ }$ & & $\sqrt{ }$ & $\sqrt{ }$ & & ( & $\sqrt{ }$ \\
\hline Kumar (2010) & $\sqrt{ }$ & $\sqrt{ }$ & $\sqrt{ }$ & $\sqrt{ }$ & $\sqrt{ }$ & & $\sqrt{ }$ & $\sqrt{ }$ & $\sqrt{ }$ & $\sqrt{ }$ & $\sqrt{ }$ & $\sqrt{ }$ & $\sqrt{ }$ & $\sqrt{ }$ & $\sqrt{ }$ & $\sqrt{ }$ & & $\sqrt{ }$ & $\sqrt{ }$ \\
\hline Obamiro (2009) & & $\sqrt{ }$ & $\sqrt{ }$ & $\sqrt{ }$ & $\sqrt{ }$ & & & & $\sqrt{ }$ & $\sqrt{ }$ & $\sqrt{ }$ & $\sqrt{ }$ & & & $\sqrt{ }$ & $\sqrt{ }$ & & & $\sqrt{ }$ \\
\hline Meybodi (2009) & & $\sqrt{ }$ & $\sqrt{ }$ & $\sqrt{ }$ & $\sqrt{ }$ & $\sqrt{ }$ & & $\sqrt{ }$ & & & $\sqrt{ }$ & $\sqrt{ }$ & $\sqrt{ }$ & $\sqrt{ }$ & & & & & \\
\hline Farooquiea et al. (2009) & & $\sqrt{ }$ & & $\sqrt{ }$ & $\sqrt{ }$ & & & $\sqrt{ }$ & $\sqrt{ }$ & $\sqrt{ }$ & $\sqrt{ }$ & & $\sqrt{ }$ & & & & & & \\
\hline Fernandez (2008) & & $\sqrt{ }$ & $\sqrt{ }$ & $\sqrt{ }$ & $\sqrt{ }$ & & $\sqrt{ }$ & & & $\sqrt{ }$ & $\sqrt{ }$ & $\sqrt{ }$ & & & $\sqrt{ }$ & & & & \\
\hline Sandanayake et al. (2008) & & $\sqrt{ }$ & $\sqrt{ }$ & $\sqrt{ }$ & $\sqrt{ }$ & & $\sqrt{ }$ & $\sqrt{ }$ & & $\sqrt{ }$ & & $\sqrt{ }$ & & & $\sqrt{ }$ & & & & $\sqrt{ }$ \\
\hline Moreira et al. (2008) & & $\sqrt{ }$ & $\sqrt{ }$ & $\sqrt{ }$ & $\sqrt{ }$ & & & $\sqrt{ }$ & $\sqrt{ }$ & $\sqrt{ }$ & $\sqrt{ }$ & $\sqrt{ }$ & & $\sqrt{ }$ & $\sqrt{ }$ & & & & \\
\hline Sendil Kumar et al. & & & & & & & $\sqrt{ }$ & & & & & & & & & & & & \\
\hline Abdallah et al. (2007) & & & & & & $\sqrt{ }$ & $\sqrt{ }$ & & $\sqrt{ }$ & & & & $\sqrt{ }$ & & $\sqrt{ }$ & & & & \\
\hline Younies et al. (2007) & & $\sqrt{ }$ & & $\sqrt{ }$ & & & & & & $\sqrt{ }$ & $\sqrt{ }$ & $\sqrt{ }$ & & & & & & & \\
\hline Wakchaure et al. (2006) & $\sqrt{ }$ & $\sqrt{ }$ & $\sqrt{ }$ & $\sqrt{ }$ & $\sqrt{ }$ & $\sqrt{ }$ & $\sqrt{ }$ & $\sqrt{ }$ & $\sqrt{ }$ & $\sqrt{ }$ & $\sqrt{ }$ & $\sqrt{ }$ & & $\sqrt{ }$ & $\sqrt{ }$ & $\sqrt{ }$ & $\sqrt{ }$ & $\sqrt{ }$ & $\sqrt{ }$ \\
\hline \multicolumn{20}{|l|}{ Wong et al. (2006) } \\
\hline Salaheldin (2005) & & $\sqrt{ }$ & $\sqrt{ }$ & $\sqrt{ }$ & & & & & & $\sqrt{ }$ & & $\sqrt{ }$ & $\sqrt{ }$ & & $\sqrt{ }$ & $\sqrt{ }$ & & & \\
\hline Phan et al. (2005) & & $\sqrt{ }$ & & & & $\sqrt{ }$ & $\sqrt{ }$ & & & & & & & & $\sqrt{ }$ & & $\sqrt{ }$ & & $\sqrt{ }$ \\
\hline Yang \& Pan (2004) & & & & & & $\sqrt{ }$ & & & & & & & & & $\sqrt{ }$ & $\sqrt{ }$ & $\sqrt{ }$ & & \\
\hline Kumar et al. (2004) & $\sqrt{ }$ & $\sqrt{ }$ & $\sqrt{ }$ & $\sqrt{ }$ & $\sqrt{ }$ & $\sqrt{ }$ & $\sqrt{ }$ & $\sqrt{ }$ & $\sqrt{ }$ & $\sqrt{ }$ & $\sqrt{ }$ & $\sqrt{ }$ & & & $\sqrt{ }$ & $\sqrt{ }$ & $\sqrt{ }$ & $\sqrt{ }$ & $\sqrt{ }$ \\
\hline Yasin et al. (2004) & & & & & & & & $\sqrt{ }$ & & & & $\sqrt{ }$ & $\sqrt{ }$ & & $\sqrt{ }$ & $\sqrt{ }$ & $\sqrt{ }$ & & \\
\hline Low et al. (2003) & & $\sqrt{ }$ & & & & & $\sqrt{ }$ & & & & & $\sqrt{ }$ & $\sqrt{ }$ & & & & & & \\
\hline Hopp et al. (2003) & $\sqrt{ }$ & $\sqrt{ }$ & & & $\sqrt{ }$ & & $\sqrt{ }$ & $\sqrt{ }$ & & & & & & & & & & & \\
\hline Kaynak (2002) & & $\sqrt{ }$ & $\sqrt{ }$ & $\sqrt{ }$ & & & & & $\sqrt{ }$ & & $\sqrt{ }$ & $\sqrt{ }$ & $\sqrt{ }$ & & & & & & \\
\hline Chung et al. (2001) & & $\sqrt{ }$ & $\sqrt{ }$ & & & & & & & & & & & & $\sqrt{ }$ & & & & \\
\hline Fullerton et al. (2001) & & & $\sqrt{ }$ & & & & $\sqrt{ }$ & & $\sqrt{ }$ & $\sqrt{ }$ & & & & & $\sqrt{ }$ & & & & \\
\hline Sui Pheng et al. (2001) & $\sqrt{ }$ & & & & & & & & & & $\sqrt{ }$ & & & & & & & & \\
\hline White et al. (2001) & & $\sqrt{ }$ & $\sqrt{ }$ & $\sqrt{ }$ & & & $\sqrt{ }$ & & $\sqrt{ }$ & $\sqrt{ }$ & $\sqrt{ }$ & $\sqrt{ }$ & & & $\sqrt{ }$ & & & & \\
\hline \multicolumn{20}{|l|}{ Sui Pheng et al. (2001a) } \\
\hline Canel et al. (2000) & & & & & & $\sqrt{ }$ & & & & & & $\sqrt{ }$ & & & & & & & $\sqrt{ }$ \\
\hline Garg \& Deshmukh (1999) & & $\sqrt{ }$ & & $\sqrt{ }$ & $\sqrt{ }$ & $\sqrt{ }$ & & $\sqrt{ }$ & & & $\sqrt{ }$ & $\sqrt{ }$ & & & $\sqrt{ }$ & & & & $\sqrt{ }$ \\
\hline Gelinas (1999) & $\sqrt{ }$ & $\sqrt{ }$ & $\sqrt{ }$ & $\sqrt{ }$ & & & & & & $\sqrt{ }$ & $\sqrt{ }$ & $\sqrt{ }$ & $\sqrt{ }$ & & $\sqrt{ }$ & & & & \\
\hline Lorefice (1998) & & $\sqrt{ }$ & & & & & $\sqrt{ }$ & & & & & & & & $\sqrt{ }$ & & $\sqrt{ }$ & & \\
\hline Gunasekaran \& Lyu & & $\sqrt{ }$ & & & & & & & & $\sqrt{ }$ & & & & & & & & & $\sqrt{ }$ \\
\hline Sakakibara et al. (1997) & & & & & & & $\sqrt{ }$ & & $\sqrt{ }$ & $\sqrt{ }$ & & & & & $\sqrt{ }$ & $\sqrt{ }$ & & & \\
\hline McLachlin (1997) & & $\sqrt{ }$ & $\sqrt{ }$ & $\sqrt{ }$ & $\sqrt{ }$ & & & & & $\sqrt{ }$ & $\sqrt{ }$ & $\sqrt{ }$ & $\sqrt{ }$ & & $\sqrt{ }$ & & $\sqrt{ }$ & & \\
\hline Mahadevan (1997) & & $\sqrt{ }$ & $\sqrt{ }$ & $\sqrt{ }$ & & & $\sqrt{ }$ & & $\sqrt{ }$ & $\sqrt{ }$ & $\sqrt{ }$ & $\sqrt{ }$ & & & $\sqrt{ }$ & & $\sqrt{ }$ & $\sqrt{ }$ & \\
\hline Gelinas et al. (1996) & & $\sqrt{ }$ & & & & & & & & & & & $\sqrt{ }$ & & & & & & \\
\hline Zhu et al. (1994) & & & $\sqrt{ }$ & $\sqrt{ }$ & & & & & & $\sqrt{ }$ & $\sqrt{ }$ & $\sqrt{ }$ & & & $\sqrt{ }$ & $\sqrt{ }$ & $\sqrt{ }$ & & \\
\hline Chen et al. (1994) & & & & & & & & $\sqrt{ }$ & $\sqrt{ }$ & $\sqrt{ }$ & & $\sqrt{ }$ & & & $\sqrt{ }$ & $\sqrt{ }$ & $\sqrt{ }$ & & \\
\hline Ramarapu et al. (1994) & & $\sqrt{ }$ & $\sqrt{ }$ & $\sqrt{ }$ & & $\sqrt{ }$ & & $\sqrt{ }$ & & $\sqrt{ }$ & $\sqrt{ }$ & $\sqrt{ }$ & & & $\sqrt{ }$ & $\sqrt{ }$ & $\sqrt{ }$ & & $\sqrt{ }$ \\
\hline Ebrahimpour et al. (1993) & & $\sqrt{ }$ & $\sqrt{ }$ & $\sqrt{ }$ & $\sqrt{ }$ & & & $\sqrt{ }$ & $\sqrt{ }$ & $\sqrt{ }$ & $\sqrt{ }$ & $\sqrt{ }$ & $\sqrt{ }$ & & $\sqrt{ }$ & & & & \\
\hline Sewell (1990) & & & & & $\sqrt{ }$ & & $\sqrt{ }$ & & $\sqrt{ }$ & & & & & & $\sqrt{ }$ & $\sqrt{ }$ & & & \\
\hline M. Reda (1987) & & & & & & & $\sqrt{ }$ & & $\sqrt{ }$ & & $\sqrt{ }$ & & & & $\sqrt{ }$ & & $\sqrt{ }$ & & $\sqrt{ }$ \\
\hline Frequency & 10 & 44 & 37 & 40 & 19 & 13 & 29 & 20 & 32 & 34 & 33 & 46 & 27 & 10 & 41 & 22 & 24 & 6 & 20 \\
\hline
\end{tabular}


Table 1

Yields literature revealed JIT elements of manufacturing (Continued)

\begin{tabular}{|c|c|c|c|c|c|c|c|c|c|c|c|c|c|c|c|c|c|c|c|}
\hline \multirow[b]{2}{*}{ Author } & \multicolumn{19}{|c|}{ JIT Elements } \\
\hline & $\mathrm{T}$ & $\mathrm{U}$ & $\mathrm{V}$ & W & $\mathrm{X}$ & $\mathrm{Y}$ & $\mathrm{Z}$ & AA & $\mathrm{AB}$ & $\mathrm{AC}$ & $\mathrm{AD}$ & $\mathrm{AE}$ & $\mathrm{AF}$ & $\mathrm{AG}$ & $\mathrm{AH}$ & AI & AJ & AK & AL \\
\hline Kumar (2014) & $\sqrt{ }$ & $\sqrt{ }$ & $\sqrt{ }$ & $\sqrt{ }$ & $\sqrt{ }$ & $\sqrt{ }$ & $\sqrt{ }$ & $\sqrt{ }$ & $\sqrt{ }$ & $\sqrt{ }$ & & & & & & & & & \\
\hline Khan (2014) & & & $\sqrt{ }$ & & & $\sqrt{ }$ & & & & & & $\sqrt{ }$ & & $\sqrt{ }$ & & & & & \\
\hline Rahmani et al. & & $\sqrt{ }$ & $\sqrt{ }$ & & $\sqrt{ }$ & & & $\sqrt{ }$ & $\sqrt{ }$ & & $\sqrt{ }$ & $\sqrt{ }$ & $\sqrt{ }$ & $\sqrt{ }$ & $\sqrt{ }$ & & & & \\
\hline Kamboj et al. (2013) & & & & $\sqrt{ }$ & $\sqrt{ }$ & $\sqrt{ }$ & & & $\sqrt{ }$ & & & & & & & & & & \\
\hline Paliwal et al. (2013) & & $\sqrt{ }$ & & $\sqrt{ }$ & $\sqrt{ }$ & $\sqrt{ }$ & & $\sqrt{ }$ & & & $\sqrt{ }$ & & $\sqrt{ }$ & $\sqrt{ }$ & & $\sqrt{ }$ & $\sqrt{ }$ & $\sqrt{ }$ & \\
\hline Kootanaee et al. & & & & $\sqrt{ }$ & $\sqrt{ }$ & $\sqrt{ }$ & & & $\sqrt{ }$ & $\sqrt{ }$ & & & $\sqrt{ }$ & & $\sqrt{ }$ & & & & \\
\hline Fateha et al. (2012) & $\sqrt{ }$ & $\sqrt{ }$ & $\sqrt{ }$ & $\sqrt{ }$ & $\sqrt{ }$ & & & $\sqrt{ }$ & & & $\sqrt{ }$ & $\sqrt{ }$ & $\sqrt{ }$ & $\sqrt{ }$ & $\sqrt{ }$ & $\sqrt{ }$ & $\sqrt{ }$ & $\sqrt{ }$ & $\sqrt{ }$ \\
\hline Gupta (2012) & & $\sqrt{ }$ & $\sqrt{ }$ & & $\sqrt{ }$ & $\sqrt{ }$ & & & & & $\sqrt{ }$ & $\sqrt{ }$ & $\sqrt{ }$ & & & & $\sqrt{ }$ & & \\
\hline Farsijani et al. (2012) & & $\sqrt{ }$ & $\sqrt{ }$ & & $\sqrt{ }$ & $\sqrt{ }$ & & $\sqrt{ }$ & & & $\sqrt{ }$ & $\sqrt{ }$ & $\sqrt{ }$ & $\sqrt{ }$ & & & & $\sqrt{ }$ & $\sqrt{ }$ \\
\hline Gupta \& Garg (2012) & & & & $\sqrt{ }$ & $\sqrt{ }$ & & & & $\sqrt{ }$ & $\sqrt{ }$ & & & $\sqrt{ }$ & & & $\sqrt{ }$ & & $\sqrt{ }$ & \\
\hline Gupta (2012) & & $\sqrt{ }$ & & $\sqrt{ }$ & $\sqrt{ }$ & $\sqrt{ }$ & & & $\sqrt{ }$ & $\sqrt{ }$ & $\sqrt{ }$ & & $\sqrt{ }$ & & & & & $\sqrt{ }$ & $\sqrt{ }$ \\
\hline Fouad et al. (2012) & $\sqrt{ }$ & $\sqrt{ }$ & $\sqrt{ }$ & & $\sqrt{ }$ & & $\sqrt{ }$ & $\sqrt{ }$ & & $\sqrt{ }$ & & & $\sqrt{ }$ & $\sqrt{ }$ & & $\sqrt{ }$ & & $\sqrt{ }$ & $\sqrt{ }$ \\
\hline Singh et al. (2012) & & $\sqrt{ }$ & $\sqrt{ }$ & $\sqrt{ }$ & $\sqrt{ }$ & $\sqrt{ }$ & $\sqrt{ }$ & $\sqrt{ }$ & & $\sqrt{ }$ & $\sqrt{ }$ & $\sqrt{ }$ & & $\sqrt{ }$ & & & & & \\
\hline Shah (2011) & & & & & & & & & & & & & & $\sqrt{ }$ & & $\sqrt{ }$ & $\sqrt{ }$ & & \\
\hline Singh et al. (2011) & $\sqrt{ }$ & $\sqrt{ }$ & & & $\sqrt{ }$ & $\sqrt{ }$ & & $\sqrt{ }$ & $\sqrt{ }$ & $\sqrt{ }$ & $\sqrt{ }$ & & $\sqrt{ }$ & $\sqrt{ }$ & & $\sqrt{ }$ & $\sqrt{ }$ & $\sqrt{ }$ & $\sqrt{ }$ \\
\hline Lohar (2011) & & & $\sqrt{ }$ & & & $\sqrt{ }$ & & $\sqrt{ }$ & & & $\sqrt{ }$ & & $\sqrt{ }$ & $\sqrt{ }$ & & $\sqrt{ }$ & $\sqrt{ }$ & & \\
\hline Singh (2011) & & $\sqrt{ }$ & & & $\sqrt{ }$ & & & & $\sqrt{ }$ & $\sqrt{ }$ & & & $\sqrt{ }$ & & & $\sqrt{ }$ & $\sqrt{ }$ & & \\
\hline Gupta (2011) & $\sqrt{ }$ & & $\sqrt{ }$ & & & & & & & & & & $\sqrt{ }$ & & & & & $\sqrt{ }$ & $\sqrt{ }$ \\
\hline Tewari et al. (2011) & & & & $\sqrt{ }$ & & & & $\sqrt{ }$ & & & & & $\sqrt{ }$ & & & $\sqrt{ }$ & & & \\
\hline Pheng et al. (2011) & & $\sqrt{ }$ & & & $\sqrt{ }$ & & & & & & $\sqrt{ }$ & $\sqrt{ }$ & $\sqrt{ }$ & & & & & & \\
\hline Gupta (2011) & $\sqrt{ }$ & $\sqrt{ }$ & & $\sqrt{ }$ & $\sqrt{ }$ & $\sqrt{ }$ & & $\sqrt{ }$ & $\sqrt{ }$ & $\sqrt{ }$ & $\sqrt{ }$ & & $\sqrt{ }$ & & & $\sqrt{ }$ & 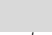 & $\sqrt{ }$ & $\sqrt{ }$ \\
\hline Hou et al. (2011) & $\sqrt{ }$ & $\sqrt{ }$ & $\sqrt{ }$ & & $\sqrt{ }$ & & & $\sqrt{ }$ & & & & $\sqrt{ }$ & & $\sqrt{ }$ & & $\sqrt{ }$ & $\sqrt{ }$ & $\sqrt{ }$ & $\sqrt{ }$ \\
\hline Agrawal (2010) & $\sqrt{ }$ & $\sqrt{ }$ & & & $\sqrt{ }$ & & & & & & $\sqrt{ }$ & $\sqrt{ }$ & & $\sqrt{ }$ & & $\sqrt{ }$ & $\sqrt{ }$ & $\sqrt{ }$ & $\sqrt{ }$ \\
\hline Mackelprang \& Nair & & & & & $\sqrt{ }$ & $\sqrt{ }$ & $\sqrt{ }$ & $\sqrt{ }$ & $\sqrt{ }$ & & $\sqrt{ }$ & & $\sqrt{ }$ & & & $\sqrt{ }$ & $\sqrt{ }$ & & \\
\hline Adeyemi (2010) & $\sqrt{ }$ & $\sqrt{ }$ & & & & $\sqrt{ }$ & & $\sqrt{ }$ & $\sqrt{ }$ & & $\sqrt{ }$ & & $\sqrt{ }$ & $\sqrt{ }$ & & $\sqrt{ }$ & $\sqrt{ }$ & $\sqrt{ }$ & $\sqrt{ }$ \\
\hline Kumar (2010) & $\sqrt{ }$ & $\sqrt{ }$ & & & $\sqrt{ }$ & $\sqrt{ }$ & $\sqrt{ }$ & $\sqrt{ }$ & $\sqrt{ }$ & & $\sqrt{ }$ & $\sqrt{ }$ & $\sqrt{ }$ & & & $\sqrt{ }$ & & $\sqrt{ }$ & $\sqrt{ }$ \\
\hline Obamiro (2009) & $\sqrt{ }$ & $\sqrt{ }$ & & & $\sqrt{ }$ & & & $\sqrt{ }$ & & & $\sqrt{ }$ & & & & & & 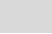 & $\sqrt{ }$ & $\sqrt{ }$ \\
\hline Meybodi (2009) & & $\sqrt{ }$ & $\sqrt{ }$ & $\sqrt{ }$ & $\sqrt{ }$ & $\sqrt{ }$ & $\sqrt{ }$ & & & & $\sqrt{ }$ & & & $\sqrt{ }$ & & & & & \\
\hline Farooquiea et al. & & & & $\sqrt{ }$ & $\sqrt{ }$ & & & & $\sqrt{ }$ & & & & & $\sqrt{ }$ & & & & & \\
\hline Fernandez (2008) & & & & & $\sqrt{ }$ & $\sqrt{ }$ & & $\sqrt{ }$ & $\sqrt{ }$ & $\sqrt{ }$ & $\sqrt{ }$ & & & & & & $\sqrt{ }$ & & \\
\hline Sandanayake et al. & $\sqrt{ }$ & $\sqrt{ }$ & & & $\sqrt{ }$ & $\sqrt{ }$ & & $\sqrt{ }$ & & & $\sqrt{ }$ & & $\sqrt{ }$ & $\sqrt{ }$ & & & $\sqrt{ }$ & $\sqrt{ }$ & $\sqrt{ }$ \\
\hline Moreira et al. (2008) & & & $\sqrt{ }$ & & $\sqrt{ }$ & $\sqrt{ }$ & $\sqrt{ }$ & & $\sqrt{ }$ & $\sqrt{ }$ & $\sqrt{ }$ & & $\sqrt{ }$ & & & $\sqrt{ }$ & $\sqrt{ }$ & $\sqrt{ }$ & $\sqrt{ }$ \\
\hline Sendil Kumar et al. & & & & & & & & $\sqrt{ }$ & & & & & & & & & & & \\
\hline Abdallah et al. (2007) & & & $\sqrt{ }$ & & & $\sqrt{ }$ & & $\sqrt{ }$ & & & & & & $\sqrt{ }$ & & & $\sqrt{ }$ & & \\
\hline Younies et al. (2007) & & & & & $\sqrt{ }$ & & & & & & & & & & & & & & \\
\hline Wakchaure et al. & $\sqrt{ }$ & $\sqrt{ }$ & & $\sqrt{ }$ & $\sqrt{ }$ & $\sqrt{ }$ & $\sqrt{ }$ & $\sqrt{ }$ & $\sqrt{ }$ & $\sqrt{ }$ & $\sqrt{ }$ & $\sqrt{ }$ & $\sqrt{ }$ & $\sqrt{ }$ & $\sqrt{ }$ & $\sqrt{ }$ & $\sqrt{ }$ & $\sqrt{ }$ & $\sqrt{ }$ \\
\hline Wong et al. (2006) & & & & & & $\sqrt{ }$ & & $\sqrt{ }$ & & & & & & & & & & & \\
\hline Salaheldin (2005) & & $\sqrt{ }$ & $\sqrt{ }$ & & $\sqrt{ }$ & & & & & & & $\sqrt{ }$ & $\sqrt{ }$ & $\sqrt{ }$ & & & $\sqrt{ }$ & $\sqrt{ }$ & \\
\hline Phan et al. (2005) & & & & & & $\sqrt{ }$ & & & & & & & & & & & $\sqrt{ }$ & $\sqrt{ }$ & \\
\hline Yang \& Pan (2004) & & & & & & & & & & & & & & & & & $\sqrt{ }$ & & \\
\hline Kumar et al. (2004) & $\sqrt{ }$ & $\sqrt{ }$ & & $\sqrt{ }$ & $\sqrt{ }$ & $\sqrt{ }$ & $\sqrt{ }$ & $\sqrt{ }$ & $\sqrt{ }$ & $\sqrt{ }$ & $\sqrt{ }$ & & $\sqrt{ }$ & & & & $\sqrt{ }$ & $\sqrt{ }$ & \\
\hline Yasin et al. (2004) & & $\sqrt{ }$ & $\sqrt{ }$ & & $\sqrt{ }$ & $\sqrt{ }$ & & $\sqrt{ }$ & $\sqrt{ }$ & $\sqrt{ }$ & $\sqrt{ }$ & & $\sqrt{ }$ & & & & & & \\
\hline Low et al. (2003) & & $\sqrt{ }$ & & $\sqrt{ }$ & $\sqrt{ }$ & & & & $\sqrt{ }$ & & $\sqrt{ }$ & $\sqrt{ }$ & $\sqrt{ }$ & & & & & & \\
\hline Hopp et al. (2003) & & $\sqrt{ }$ & & & $\sqrt{ }$ & $\sqrt{ }$ & & $\sqrt{ }$ & $\sqrt{ }$ & $\sqrt{ }$ & $\sqrt{ }$ & & & & $\sqrt{ }$ & $\sqrt{ }$ & $\sqrt{ }$ & $\sqrt{ }$ & \\
\hline Kaynak (2002) & & $\sqrt{ }$ & $\sqrt{ }$ & & $\sqrt{ }$ & & & & & $\sqrt{ }$ & $\sqrt{ }$ & & $\sqrt{ }$ & $\sqrt{ }$ & & $\sqrt{ }$ & $\sqrt{ }$ & $\sqrt{ }$ & \\
\hline Chung et al. (2001) & & & $\sqrt{ }$ & & & & & & & $\sqrt{ }$ & & & & $\sqrt{ }$ & $\sqrt{ }$ & & $\sqrt{ }$ & & \\
\hline Fullerton et al. (2001) & $\sqrt{ }$ & $\sqrt{ }$ & & $\sqrt{ }$ & & & & & $\sqrt{ }$ & $\sqrt{ }$ & $\sqrt{ }$ & & $\sqrt{ }$ & $\sqrt{ }$ & & & $\sqrt{ }$ & $\sqrt{ }$ & $\sqrt{ }$ \\
\hline Sui Pheng et al. & & & $\sqrt{ }$ & & $\sqrt{ }$ & & & & $\sqrt{ }$ & & $\sqrt{ }$ & $\sqrt{ }$ & $\sqrt{ }$ & & & & & & \\
\hline White et al. (2001) & & & $\sqrt{ }$ & & $\sqrt{ }$ & & & & $\sqrt{ }$ & & $\sqrt{ }$ & & $\sqrt{ }$ & & & & $\sqrt{ }$ & $\sqrt{ }$ & \\
\hline Sui Pheng et al. & & & & & $\sqrt{ }$ & & & & & & $\sqrt{ }$ & $\sqrt{ }$ & $\sqrt{ }$ & & & & & & \\
\hline Canel et al. (2000) & & & & & & $\sqrt{ }$ & & & $\sqrt{ }$ & & $\sqrt{ }$ & & & & & & & & \\
\hline Garg \& Deshmukh & $\sqrt{ }$ & $\sqrt{ }$ & $\sqrt{ }$ & & & $\sqrt{ }$ & $\sqrt{ }$ & $\sqrt{ }$ & & $\sqrt{ }$ & $\sqrt{ }$ & $\sqrt{ }$ & $\sqrt{ }$ & $\sqrt{ }$ & $\sqrt{ }$ & $\sqrt{ }$ & $\sqrt{ }$ & $\sqrt{ }$ & $\sqrt{ }$ \\
\hline Gelinas (1999) & $\sqrt{ }$ & $\sqrt{ }$ & $\sqrt{ }$ & & $\sqrt{ }$ & $\sqrt{ }$ & & $\sqrt{ }$ & & & & & $\sqrt{ }$ & $\sqrt{ }$ & $\sqrt{ }$ & $\sqrt{ }$ & $\sqrt{ }$ & $\sqrt{ }$ & \\
\hline Lorefice (1998) & & & & & $\sqrt{ }$ & & & $\sqrt{ }$ & & & $\sqrt{ }$ & & & & & & $\sqrt{ }$ & $\sqrt{ }$ & $\sqrt{ }$ \\
\hline Gunasekaran \& Lyu & & & & & & $\sqrt{ }$ & & & & & $\sqrt{ }$ & & & & & & & & \\
\hline Sakakibara et al. & & & $\sqrt{ }$ & & & $\sqrt{ }$ & & $\sqrt{ }$ & & & & & $\sqrt{ }$ & $\sqrt{ }$ & & & $\sqrt{ }$ & $\sqrt{ }$ & \\
\hline McLachlin (1997) & $\sqrt{ }$ & $\sqrt{ }$ & & $\sqrt{ }$ & $\sqrt{ }$ & & & & $\sqrt{ }$ & & & & $\sqrt{ }$ & & & $\sqrt{ }$ & & $\sqrt{ }$ & \\
\hline Mahadevan (1997) & $\sqrt{ }$ & $\sqrt{ }$ & $\sqrt{ }$ & & $\sqrt{ }$ & & & $\sqrt{ }$ & & & & & $\sqrt{ }$ & $\sqrt{ }$ & & $\sqrt{ }$ & $\sqrt{ }$ & $\sqrt{ }$ & \\
\hline Gelinas et al. (1996) & & & $\sqrt{ }$ & & & & & & $\sqrt{ }$ & & & & & & & & $\sqrt{ }$ & & \\
\hline Zhu et al. (1994) & & $\sqrt{ }$ & $\sqrt{ }$ & $\sqrt{ }$ & $\sqrt{ }$ & & & $\sqrt{ }$ & $\sqrt{ }$ & & & & $\sqrt{ }$ & & & & & & \\
\hline Chen et al. (1994) & & $\sqrt{ }$ & & $\sqrt{ }$ & $\sqrt{ }$ & & & & $\sqrt{ }$ & & & & $\sqrt{ }$ & & & & & & \\
\hline Ramarapu et al. & $\sqrt{ }$ & & $\sqrt{ }$ & & & $\sqrt{ }$ & & $\sqrt{ }$ & $\sqrt{ }$ & & $\sqrt{ }$ & & $\sqrt{ }$ & & & $\sqrt{ }$ & & $\sqrt{ }$ & $\sqrt{ }$ \\
\hline Ebrahimpour et al. & & $\sqrt{ }$ & & & $\sqrt{ }$ & & & & & & $\sqrt{ }$ & & $\sqrt{ }$ & & & & & & \\
\hline Sewell (1990) & & & $\sqrt{ }$ & & $\sqrt{ }$ & $\sqrt{ }$ & & & $\sqrt{ }$ & & $\sqrt{ }$ & & & $\sqrt{ }$ & $\sqrt{ }$ & & & $\sqrt{ }$ & $\sqrt{ }$ \\
\hline M. Reda (1987) & & & & $\sqrt{ }$ & $\sqrt{ }$ & & & & $\sqrt{ }$ & & & & & & $\sqrt{ }$ & $\sqrt{ }$ & & & \\
\hline Frequency & 20 & 35 & 28 & 20 & 46 & 33 & 10 & 32 & 31 & 19 & 37 & 16 & 40 & 27 & 10 & 25 & 31 & 32 & 20 \\
\hline
\end{tabular}


Even although the JIT manufacturing system seems to be attention-grabbing and less difficult, it needs harmonization with supply chain to keep away from delays in the manufacturing schedule. Gupta (2012) reviewed literature on JIT philosophy implementation in Indian context and some research issues were highlighted. Farsijani et al. (2012) described JIT as world class production techniques that emphasizes on meeting external and internal customers' needs and expectations. The function of this reading is to introduce a mathematical method for identifying elements and components that are critical for JIT success in different circumstances. TOPSIS and Pareto techniques are used to implement JIT. We set priorities in these techniques for selection of component and then use entropy method to give coefficient to TOPSIS matrix. Gupta et al. (2012) widely discussed the JIT manufacturing philosophy in different industries of Indian context and highlighted some important insights pertaining to it. Fouad et al. (2012) evaluated the scale up to which JIT is implemented in five main industrial sectors of Middle East, such as food, construction, chemicals, fabrics and engineering and developed two main hypotheses and tested using descriptive statistics and ANOVA. Garg et al. (2013) addressed the necessary initiatives essential to be taken by the management of Indian manufacturing industries. They investigated and concluded the operations and procedures modifications required for the successful accomplishment of JIT philosophy with the assist of survey conducted on literature. Poliwal et al. (2013) explained the broadening of JIT concepts, movement, benefits, obstructions and execution of JIT philosophy in Indian industrialized system by analyzing literature. Babu et al. (2013) discussed in depth the overall JIT concepts and the factors necessary for JIT philosophy implementation in Indian context. Kumar (2014) reviewed literature associated to JIT usage and performance in Indian perspective. Thirty-seven identified elements of JIT production system were divided into five major categories twenty-one research papers and relative importance of all the thirty-seven elements were highlighted. Khan (2014) did a survey on Textile weaving industry of Karachi and highlighted important JIT drivers such as supplier selection criteria, layout of company, inventory management, scheduling, quality standard, maintenance policy, employee consideration, political volatility, power shortage, buyer consistent demand, and infrastructure and wakefulness of JIT approach in this sector is of considerable importance. Rahmani et al. (2014) reported the increase in performance of automotive industry after accomplishment of JIT. To decrease the amount of waste produced was declared as one of the greatest strategies in production.

Table 2

List of Alphabetic abbreviations used in table 1

\begin{tabular}{llll}
\hline $\begin{array}{l}\text { Alphabetic } \\
\text { abbreviation }\end{array}$ & Name of element & $\begin{array}{l}\text { Alphabetic } \\
\text { abbreviation }\end{array}$ & Name of element \\
\hline A & Buffer Stock Removal & T & Statistical Process control \\
B & Continuous Improvement (kaizen) & U & Statistical quality control \\
C & Effective Communication & V & Strong buyer-supplier relationship \\
D & Employee Empowerment \& Involvement & W & Total quality control \\
E & Error Prevention (poka-yoke) & X & Layout improvement \\
F & Frequent and Reliable delivery & Y & Vendor rating \\
G & Kanban & Z & Scheduling flexibility/ Under capacity Scheduling \\
H & Long Term Quality Control [QC] commitment & AA & Zero defect \\
I & Multi-functional worker & AB & 100\% quality inspection \\
J & Total Productive Maintenance & AC & Waste Reduction \\
K & QC authority to worker & AD & Uninterrupted work flow \\
L & Education \& training to workers \& suppliers & AE & Top Management Commitment \\
M & Quality certification of supplier & AF & Inventory Management \\
N & Regular quality auditing & AG & MRP \\
O & Set up time reduction & AH & Autonomation (Jidoka) \\
P & Short lead time & AI & JIT Purchasing \\
Q & Small lot size & AJ & Process Simplification \\
R & Standard containers & AK & Process Flexibility \\
S & Standardization & AL & \\
\hline
\end{tabular}

Fig. 1 shows the degree of importance of JIT Elements in the Literature. 


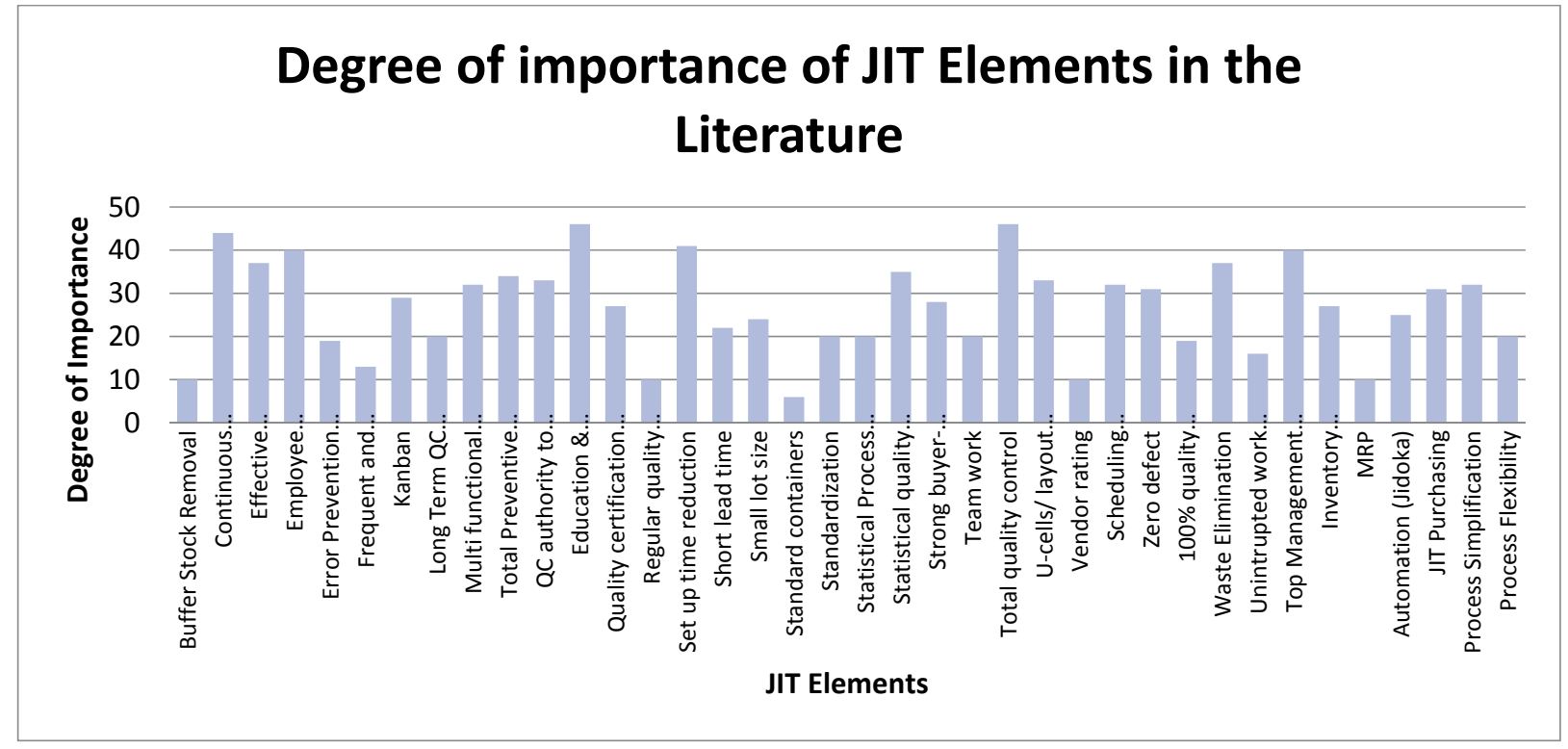

\section{JIT Elements}

Fig. 1. The degree of importance of JIT Elements in the Literature

Buffer stock removal is a JIT technique to reduce non-value added activities. A buffer stock approach is an attempt of storage to steady prices in the market. Buffer stock has strong relationship with lot size and JIT purchase, and weak relationship with process and product standardization. Inventory covers up a lot of wasteful practices. Better maintained machines, improved quality practices and delivery times, streamlined machine setup actions and efficient labor and equipment utilization permits the organization to function with less inventory and cost, and faster response times in meeting customer needs. Buffer stock is more of production/ purchase problem. Since upholding is a process in nature with standardization approach, thus buffer stock removal maintains the least effect on JIT maintenance. Continuous improvement (Kaizen) means "change for better". The word also refers to continuous. It is widely researched in production systems. There exists no processes that are perfect, thus a need for continuous improvement is always there. Companies applying this philosophy have gained great economic benefits. Maintenance is an activity to modify for better and it requires continuous improvement. A maintenance plan should include strategies for continuous improvement, since plan is totally perfect; it should be improved. Thus, kaizen desires to be measured as a JIT element for maintenance. Effective communication in JIT aims in time and material waste reduction. And maintenance is an activity for which time is more valuable. The maintenance time needs to be minimized. Thus, for effective utilization of maintenance time effective communication have a pilot role. Effective communication between different departments (especially purchase and inventory) and among the maintenance personals is always beneficial for the growth of the industries. It is essential to understand what actually one wants to express, thus effective communication is necessary activity of maintenance. The communication medium and language has a great impact on maintenance activity. Employee empowerment and involvement is a necessary activity.

Victorious implementation of JIT in an organization largely depends on front-line employees. Their role is to collaboratively work with higher and lower levels of hierarchy. Their role is to work for improvement of work processes, understand quality measures, solve problems, generate broader outlook of the production process, ensure inter-connectivity of workers and decide basic maintenance practices, to understand the condition of machines and equipment's and ability to meet quality and production requirements. Thus, employee empowerment and involvement is the key in element of maintenance practice, and desires to be measured as JIT element for maintenance. Error prevention (poke-yoke) is an apparatus for inadvertent error prevention and to prevent errors originating in the 
mistake to reduce quality control. Application of poke yoke in maintenance will reduce breakdown and improve the accessibility of machines as it aims to eliminate possibility of the error occurrence. Frequent and reliable delivery is desirable from any manufacturing unit. The reliability of the delivery depends on the accessibility of the machines and equipment. The availability in turn is maintained by reduced uncertainty. Thus, maintenance is at third level of importance for frequent and reliable delivery. Kanban is the scheduling system for JIT and lean manufacturing. It is also an inventory control tool for supply chain. Kanban is an information system. One could also consider that Kanban is a very efficient tool to ensure proper delivery of information in the making line and, thus, avoid mistakes.

It is an effective tool to support manufacturing. Thus, the scheduling tool is of less importance to maintenance. Long term QC commitment is not an overnight achievement. It is the consequence of close agreement of JIT principles with hard work over many quality plans. It can happen when the organization has the least uncertainty. It requires each worker to organize the quality of the processes carried out. Both JIT and quality are philosophies applied to production systems, and philosophies are not implemented within a little time since they must be integrated into the lifestyle of workers. A longterm quality plan helps to generate strategies to remove waste in the manufacturing system, which would increase fluidity of materials. Multi-functional worker refers to the capability of the workers to execute various activities. In case of unavailability of any worker, other could perform the required task. The work remains unaffected. It was measured to be the highly useful JIT element by Jurado et al. (2014) for the success of JIT philosophy, hence considered as JIT element in maintenance. Total Preventive Maintenance is helpful to improve the quality, flexibility and cost by increasing precise time bound enhancement action plans. It is considered essential to obtain proper performance and efficiency indicators in companies.

The action plan is passed out prior to the breakdown occurs and thus the JIT element is required for JIT element in maintenance. Quality control authority to worker: This element of JIT provides authority to the workers to take counteractive action to improve the quality of the product. Since the activities during the manufacturing process add worth to a product to obtain the desired characteristics, quality desires to be analyzed through the production process. If mistakes occur during any stage of the production; the product gets affected and requires additional activities to get it corrected. The element desires to be measured for maintenance to ensure proper and in-time maintenance. Education and training of workers must be closely linked because the product quality depends not only on machinery and equipment, but also on the education and training of managers who develop plans and workers and execute these plans (Ooi, 2014). There exists a well-built bond between motivation and the educational levels of operators. Set up time lessening is required to enlarge the production rate and maximize the profits. The programs to reduce setup times for machines and equipment are based on both the analysis of unnecessary movements by operators and machines and the design of these machines. It is significant to highlight that in order to gather short preparation times highly trained and skilled working personals are required.

Standardization: It is one of the essential elements required for successful execution of JIT. By applying the perception of standardization, easy availability and inter-changeability of tools and parts are ensured, making the processes simple. Statistical Process control plays an explanation role in the success of the JIT program. It controls the process through performance indices and is equally important in all phases and departments of a company or industry. Statistical quality control: the system generated statistics are the real performance indicators of the organization status. Statistical quality control is a snapshot of health of companies. Appropriate quality control plans and programs with their application by statistical quality control are crucial for JIT implementation. Thus, the element desires to be measured for maintenance. Total quality control: Quality is a major element to reduce uncertainty and non-value-added activity in an organization. JIT concept for quality is "Do it right the first time". It eliminates time and materials waste. Upholding is a time dependent activity, thus the quality control element is strongly recommended JIT manufacturing element to be measured as JIT maintenance element. Total quality control as a JIT element for maintenance includes selecting the right person for 
the right maintenance activity, education and training of maintenance personals, equipping the maintenance team with special and updated tools and techniques, giving valuable consideration to the feedback of maintenance personals, encourage computer assisted maintenance management system, need to work for error prevention instead of error detection.

Quality certification of supplier helps in supplies selection for future use. Regular quality auditing helps to improve quality and list out the weaknesses and strengths in the system. Short lead time is the outcome by application of JIT. Small lot size reduces complexity. A standard container helps in easy counting, assembly processes and inventory handling. Layout improvement involves futuristic plans. Vendor rating identifies the best vendors and starts a contest among vendors to supply quality items and service. Scheduling flexibility/under capacity scheduling reduces complexity and ensures on time delivery. Zero defect targets improve quality. $100 \%$ quality inspection ensures no rejection and no customer complaints. Waste elimination is the key feature and element of JIT inside all business functions. Uninterrupted work flow increases the accessibility of the machines and the system. Top management assurance works like a soul in the human resources. Inventory management is again the primary element of JIT. MRP is an intelligent approach for resources management. Automation improves quality, reduces rejections and increases productivity. Process simplification is required for easy working. Process flexibility ensures on time deliverance and shorter make span. JIT purchasing decreases inventory. Leveling of production improves processes functions and workforces. Pull system reduces inventory. Strong buyer-supplier relationship develops confidence in entrepreneur for higher investment for the project. Team work improves the processes in all respects. Low cost is the desire of everyone connected to the manufactured goods. From the above listed JIT elements, the following elements are selected as JIT elements for application in maintenance. Based on the described in depth theoretical analysis of the reviewed literature and brain storming with maintenance managers/engineers of JIT elements implemented industries the following eighteen JIT elements are selected from thirtyeight JIT elements to analyze implementation of JIT in maintenance sector. Table 3 shows Critical JIT elements in implementation of JIT in Maintenance and their role.

Table 3

Critical JIT elements in JIT implementation and their roles

\begin{tabular}{ll}
\hline Critical JIT elements in implementation of JIT in Maintenance & Role of element \\
\hline Continuous Improvement (kaizen) & To improve machine availability and decrease maintenance time. \\
Effective Communication & To coordinate among the team and with others for perfect maintenance. \\
Employee Involvement & To improve involvement and skills. \\
Flexible/Multi Skilled Workforce & $\begin{array}{l}\text { Even in non-availability of skilled human resource, the work should not get } \\
\text { effected and to improve workforce flexibility. }\end{array}$ \\
Total Productive Maintenance & To reduce breakdown maintenance. \\
Workers centered quality control & Improve responsibility and work quality. \\
Education \& training & Employee remains updated with latest technological changes in maintenance. \\
Set up time reduction & Increase production by reducing time. \\
Statistical quality control & Learn from past experience. \\
Total quality control & Reduce rejections. \\
Layout improvement & Reduce complexity \\
Under capacity Scheduling & Improve workforce flexibility. \\
Zero defects & To reduce scrap and re-work tasks. \\
Waste Reduction & Reduce cost of maintenance. \\
Role of top management in maintenance & Enhance feelings of responsibility among workforce. \\
Autonomation (Jidoka) & Improves quality. \\
JIT Purchasing & Reduces inventory cost. \\
Process Simplification & Reduces maintenance time and cost. \\
\hline
\end{tabular}

\section{Conclusion}

In depth theoretical analysis of the reviewed literature is carried out after reviewing sixty-five studies related to the JIT philosophy implemented manufacturing systems of different kinds of productions. Personal interview was conducted of the maintenance managers and engineers JIT elements 
implemented Indian manufacturing organizations. Based on this analysis from the thirty-eight JIT elements, eighteen JIT elements are selected for the JIT implementation in maintenance.

\section{References}

Abdallah, A. B., \& Matsui, Y. (2007). The relationship between JIT production and Manufacturing strategy and their impact on JIT performance.

Adeyemi, S. L. (2010). JIT production systems (JITPS) in developing countries: the Nigerian experience. J Soc Sci, 22(2), 145-152.

Agrawal, N. (2010). Review on just in time techniques in manufacturing systems. Advances in Production Engineering \& Management, 5(2), 101-110.

Al, R. H. F. S. D. (2012). Executive Manager's Opinion about JIT Implementation Status in the Middle East Industry. Global Journal of Research In Engineering, 12(1-G).

Canel, C., Rosen, D., \& Anderson, E. A. (2000). JITis not just for manufacturing: a service perspective. Industrial Management \& Data Systems, 100(2), 51-60.

Chen, I. J., Chung, C. S., \& Gupta, A. (1994). The integration of JIT and FMS: issues and decisions. Integrated manufacturing systems, 5(1), 4-13.

Chung, C. K., \& Bakar, N. A. (2001). The relevancy of JIT(JIT) concept in government purchasing. Journal of Technology, 35(A), 1-8.

Ebrahimpour, M., \& Withers, B. E. (1993). A comparison of manufacturing management in JIT and non-JIT firms. International journal of production economics, 32(3), 355-364.

Ericsson, J. (1997). Störningsanalys av tillverkningssystem (Doctoral dissertation, Doctoral thesis, Lunds Tekniska Högskola, Lund, Sweden).

Faria, J., \& Nunes, E. (2012). Reliability engineering of large jit production systems. International Journal of Reliability, Quality and Safety Engineering, 19(03), 1250011.

Farooquiea, J. A., \& Mohapatrab, A. B. (2009). Japanese Manufacturing Techniques and Practices: An Indian Perspective. EDITORIAL BOARD SUPPORT TEAM, 3(3), 174.

Farsijani, H., Fard, Y. S., Kharazian, M. A., \& Nikabadi, M. S. (2012). A Method for Identifying Critical Success Factors of JIT Implementation in Different Circumstances. Journal of Supply Chain Management Systems, 1(1), 1.

Fateha, A. N., Nafrizuan, M. Y., \& Razlan, A. (2012). Review on elements of JIT implementation. In Proceedings of the International Conference on Automotive, Mechanical and Materials Engineering (ICAMME'12).

Fernández, J. L., (2008), Just in Time article at IE Business school, published by IE Publishing Department. María de Molina 13, 28006 - Madrid, Spain.

Fullerton, R. R., \& McWatters, C. S. (2001). The production performance benefits from JIT implementation. Journal of operations management, 19(1), 81-96.

Garg, D. \& Deshmukh, S. (1999). JIT purchasing: literature review and implications for Indian industry. Production Planning \& Control, 10(3), 276-285.

Gélinas, R. (1999). The JITimplementation project. International Journal of Project Management, 17(3), 171-179.

Gélinas, R., Jacob, R., \& Drolet, J. (1996). JIT purchasing and the partnership strategy. European Journal of Purchasing \& Supply Management, 2(1), 39-45.

Gunasekaran, A., \& Lyu, J. (1997). Implementation of JIT in a small company: a case study. Production planning \& control, 8(4), 406-412.

Gupta, A. K. (2012). Just in time revisited: literature review and agenda for future research. International Journal of Research in Mechanical Engineering \& Technology, 2(1), 969971.

Gupta, A. K. (2012a) JIT in Higher Education. International Journal of Research in Engineering \& Technology, 2(1), 70-73.

Gupta, R., \& Garg, D. (2012),Just in Time-A Concept for Efficient Manufacturing. International 
Journal of Applied Engineering Research, 7(11).

Gupta, A.K. (2011). JIT in Indian Context: Literature Review and Directions for Future Research. International Journal of Advances in Engineering Research, 1.

Gupta, A. K. (2011a). A conceptual JIT model of service quality. Int J Eng Sci Technol, 3(3), 22142227.

Hopp, W. J., \& Spearman, M. L. (2004). To pull or not to pull: what is the question?. Manufacturing \& service operations management, 6(2), 133-148.

Hou, B., Chan, H. K., \& Wang, X. (2011). A Case Study of JIT System in the Chinese Automotive Industry. In Proceedings of the World Congress on Engineering (Vol. 1, pp. 6-8).

Ismail Salaheldin, S. (2005). JIT implementation in Egyptian manufacturing firms: some empirical evidence. International Journal of Operations \& Production Management, 25(4), 354-370.

Javadian Kootanaee, A., Babu, K. N., \& Talari, H. F. (2013). JIT Manufacturing System: From Introduction to Implement. International Journal of Economics, Business and Finance, 1(2), 7-25.

Johannesson, P., Bergman, B., Svensson, T., Arvidsson, M., Lönnqvist, Å., Barone, S., \& Maré, J. (2013). A robustness approach to reliability. Quality and Reliability Engineering International, 29(1), 17-32.

Jonsson, P. (1999). The impact of maintenance on the production process-achieving high performance. Lund University.

Kaynak, H. (2002). The relationship between JIT purchasing techniques and firm performance. IEEE Transactions on Engineering Management, 49(3), 205-217.

Khan, N. A. (2014). Impediments to JIT Implementation in Textile Industry. International Proceedings of Economics Development and Research, 69, 83.

Kumar, V. (2014), JIT Manufacturing Systems in Indian Industries: A Review. International Journal of Engineering Sciences \& Research Technology, 1(3), 8004-8016.

Kumar, S., Kamboj, G. G. A., \& Singh, M. (2013). Difficulties of JIT Implementation. International Journal on Theoretical and Applied Research in Mechanical Engineering (IJTARME), ISSN, 23193182.

Kumar, M., Tewari, P. C., \& Gupta, A. (2011). Evaluation of Just in Time Elements in Banking Sector Using ANOVA Technique. International Journal of science, Technology and Management, 2(2), 29-39.

Kumar, V. (2010). JIT based quality management: concepts and implications in Indian context. International Journal of Engineering Science and Technology, 2(1), 40-50.

Kumar, C. S., \& Panneerselvam, R. (2007). Literature review of JIT-KANBAN system. The International Journal of Advanced Manufacturing Technology, 32(3-4), 393-408.

Kumar, V., Garg, D., \& Mehta, N. P. (2004). JIT practices in Indian context: A survey. Journal of Scientific \& Industrial Research, 63, 655-662.

Lohar, B. (2011). JIT practices in Indian industries: a survey. International Journal of Computing and Corporate Research, 1(1), 1-13.

Lorefice, A. A. (1998). Just in time manufacturing: introduction and major components. New York.

Low, S. P., \& Ang, G. K. (2003). Integrating JIT and 5-S concepts for construction site management: A case study. International Journal of Construction Management, 3(1), 31-47.

Mackelprang, A. W., \& Nair, A. (2010). Relationship between JIT manufacturing practices and performance: A meta-analytic investigation. Journal of Operations Management, 28(4), 283-302.

Mahadevan, B. (1997). Are Indian companies ready for just-in-time?.Management Review, 2(2/3), 8592.

Martínez-Jurado, P. J., Moyano-Fuentes, J., \& Jerez-Gómez, P. (2014). Human resource management in lean production adoption and implementation processes: success factors in the aeronautics industry. BRQ Business Research Quarterly, 17(1), 47-68.

McLachlin, R. (1997). Management initiatives and JIT manufacturing. Journal of Operations Management, 15(4), 271-292.

Meybodi, M. Z. (2009). Benchmarking performance measures in traditional and JITcompanies. Benchmarking: An International Journal, 16(1), 88-102. 
Moreira, M. R. M. O. A., \& Alves, R. A. F. S. (2010). A study on JIT implementation in Portugal: some empirical evidence. Brazilian Journal of Operations \& Production Management, 5(1), 5-22.

Obamiro, J. K. (2009). Exploring the Relationships Between JIT Technique and Manufacturing Performance: Empirical Evidence From Selected Nigerian Firms. Manager (University of Bucharest, Faculty of Business \& Administration), (10).

Ooi, K. B. (2014). TQM: A facilitator to enhance knowledge management? A structural analysis. Expert Systems with Applications, 41(11), 5167-5179.

Paliwal, S., Varshney, A. R., \& Awana, D. S. (2013), JIT Implementation: Concepts, Benefit and Obstructions in Indian Industries.

Phan, A. C., \& Matsui Y. (2005), JIT Practices and Competitive Performance: Empirical Evidence from Japan, International Graduate School of Social Sciences, Yokohama National University, (004 $-0301)$.

RAHMANI, K., \& NAYEBI, M. A. (2014). EFFECT OF JIT IMPLEMENTATION IN IRAN AUTOMOTIVE INDUSTRY (CASE STUDY: IRAN KHODRO'S ASSEMBLY LINE 2). Indian J. Sci. Res, 7(1), 001-016.

Ramarapu, N. K., Mehra, S., \& Frolick, M. N. (1995). A comparative analysis and review of JIT "implementation" research. International Journal of Operations \& production management, 15(1), 38-49.

Ramaswamy, N. R., Selladurai V. \& Subramaniam R. S., A Study On Priority Assignment To Various Elements Of Kaizen In A Garment Unit, in 1st international conference on Logistics and supply chain management (ILSCM), 495-499.

Reda, H. M. (1987). A review of "kanban"-the Japanese "Just-In-Time" production system. Engineering Management International, 4(2), 143-150.

Sakakibara, S., Flynn, B. B., Schroeder, R. G., \& Morris, W. T. (1997). The impact of JIT manufacturing and its infrastructure on manufacturing performance. Management Science, 43(9), 1246-1257.

Sandanayake, Y. G., Oduoza, C. F., \& Proverbs, D. G. (2008). A systematic modelling and simulation approach for JIT performance optimisation. Robotics and Computer-Integrated Manufacturing, 24(6), 735-743.

Sewell, G. (1990). Management information systems for JIT production. Omega, 18(5), 491-503.

Shah, N. H. (2011). Single Supplier-Buyer Integrated Inventory Model Under Multiple JIT Delivery and Stock-Dependent Demand. Journal of Mathematical Modelling and Algorithms, 10(3), 293305.

Singh, S.(2011), JIT System: A Cultural Difference between Japan and Indian Implementation, Int. J. Res. Mech. Technol., 1(1), 52-56.

Singh, S., \& Garg, D. (2011a). Comparative analysis of Japanese JIT purchasing and traditional Indian purchasing system. International Journal of Engineering Science and Technology, 1(3), 1816-1834.

Singh, S., \& Garg, D. (2011). JIT system: concepts, benefits and motivation in Indian industries. Int J Manag Bus Stud, 1(1), 26-30.

Sinha P. K., JIT and Lean production system : A textbook of Manufacturing and Operations Management, 9.13.

Sui Pheng, L., Manzoor Arain, F., \& Wong Yan Fang, J. (2011). Applying JIT principles in the delivery and management of airport terminal buildings. Built Environment Project and Asset Management, 1(1), 104-121.

Sui Pheng, L., \& Joo Chuan, C. (2001). JIT management in precast concrete construction: a survey of the readiness of main contractors in Singapore. Integrated Manufacturing Systems, 12(6), 416-429.

Sui Pheng, L., \& Joo Chuan, C. (2001a). A study of the readiness of precasters for JIT construction. Work study, 50(4), 131-140.

Wakchaure, V. D., Venkatesh, M. A., \& Kallurkar, S. P. (2006, June). Review of JIT practices in Indian manufacturing industries. In 2006 IEEE International Conference on Management of Innovation and Technology (Vol. 2, pp. 1099-1103). IEEE. 
White, R. E., \& Pearson, J. N. (2001). JIT, system integration and customer service. International Journal of Physical Distribution \& Logistics Management, 31(5), 313-333.

Wong, W. K., Kwong, C. K., Mok, P. Y., \& Ip, W. H. (2006). Genetic optimization of JIT operation schedules for fabric-cutting process in apparel manufacture. Journal of Intelligent Manufacturing, 17(3), 341-354.

Yang, J. S., \& Pan, J. C. H. (2004). JIT purchasing: an integrated inventory model involving deterministic variable lead time and quality improvement investment. International Journal of Production Research, 42(5), 853-863.

Yasin, M. M., Wafa, M., \& Small, M. H. (2004). Benchmarking JIT: an analysis of JIT implementations in the manufacturing service and public sectors. Benchmarking: an international journal, 11(1), 7492.

Younies, H., Barhem, B., \& Hsu, C. E. (2007). A review of the Adoption of JITmethod and its effect on efficiency. Public Administration and Management, 12(2), 25.

Zhu, Z., Meredith, P. H., \& Makboonprasith, S. (1994). Defining critical elements in JIT implementation: a survey. Industrial Management \& Data Systems, 94(5), 3-10.

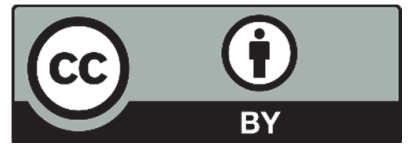

C 2017 by the authors; licensee Growing Science, Canada. This is an open access article distributed under the terms and conditions of the Creative Commons Attribution (CC-BY) license (http://creativecommons.org/licenses/by/4.0/). 